\title{
Unimodular Fourier multipliers with a time parameter on modulation spaces
}

\section{Congwei Song*}

"Correspondence:

williamzju@yahoo.com

Department of Mathematics, Zheda Road, Hangzhou, Zhejiang 310027, China

\begin{abstract}
In this paper, we mainly study the boundedness of unimodular Fourier multipliers with a time parameter $\mathrm{e}^{\mathrm{i} t p(\xi)}$ on the modulation spaces where $p(\xi)$ is a differentiable real-valued function, namely we estimate $\mathrm{e}^{\mathrm{i} t p(\xi)}$ under the multiplier norm, denoted by $\mathfrak{M}^{s, p}$. The sharpness of $s$ and the regularity lost are also discussed when the multiplier acts on functions in modulation spaces. Meanwhile the lower bound of the multiplier is shown. Finally, we present a discussion of the relationship between the main result and well-posedness results for nonlinear PDEs already existing in the literature.

MSC: Primary 42B15; 42B35; secondary 42C15
\end{abstract}

Keywords: modulation spaces; Fourier multipliers; nonlinear dispersive equations; oscillatory integrals

\section{Introduction}

The modulation spaces have been well known as the 'right' spaces in time-frequency analysis. Refer to [1-12]. Recently Hardy type modulation spaces have been proposed in [13]. In this paper, we discuss these spaces in a brief manner, and then study the Fourier multipliers on them.

\subsection{Modulation spaces}

In this subsection we will introduce modulation spaces and Wiener spaces briefly. We adopt the definitions and notation in [11] mainly.

Denote the frequency-uniform decomposition operators

$$
\square_{k} f=\mathcal{F}^{-1}\left(\psi_{k} \mathcal{F} f\right), \quad k \in \mathbb{Z}^{n}
$$

where $\psi_{k}(\xi)=\psi(\xi-k)$ constitute a so-called unity partition with good properties, and $\psi$ is a bump-like function. $M_{p, q}^{s}$ is a subspace of the distribution space $\ell^{\prime}$ with the norm

$$
\|f\|_{M_{p, q}^{s}}:=\left(\sum_{k}\langle k\rangle^{s q}\left\|\square_{k} f\right\|_{p}^{q}\right)^{\frac{1}{p}}
$$

where $\langle x\rangle=1+|x|$. Strictly speaking, only when $1 \leq p, q \leq \infty$, (1) becomes a norm. If $s=0$, we simply write $M_{p, q}$ instead of $M_{p, q}^{0}$.

The modulation spaces can be regarded as an example of the Wiener amalgam spaces $[1,14,15]$. Now we fix the definition of the class of spaces in the present paper.

○2014 Song; licensee Springer. This is an Open Access article distributed under the terms of the Creative Commons Attribution License (http://creativecommons.org/licenses/by/2.0), which permits unrestricted use, distribution, and reproduction in any medium, provided the original work is properly cited. 
Definition 1.1 Given $\psi \in \S$ as in the modulation spaces, a function Banach space $X$ and a sequence Banach space $Y$, the space $W(X, Y)$ consists of all distributions $u \in \mathcal{S}^{\prime}\left(u \in X_{\text {loc }}\right.$ precisely) for which

$$
\|u\|_{W(X, Y)}:=\left\|\left\{\left\|\psi_{k} u\right\|_{X}\right\}\right\|_{Y}<\infty
$$

Another class of spaces mentioned in the paper is

$$
\mathcal{F} X:=\{\mathscr{F} f \mid f \in X\} \text { whose norm is }\|f\|_{\mathcal{F} X}:=\|\mathcal{F} f\|_{X},
$$

where $X$ is $L^{p}$ or any other known space for which $\mathcal{F} f, f \in X$, makes sense. Actually, $M_{p, q}^{s}=$ $\mathcal{F} W\left(\mathcal{F} L^{p}, \ell^{s, q}\right)$, where $\ell^{s, q}$ is the $q$-summable sequence with the weight $\langle k\rangle^{s}$. Moreover, Hardy type modulation spaces $\mu_{p, q}^{s}=\mathscr{F} W\left(\mathcal{F} H^{p}, \ell^{s, q}\right), 0<p \leq 1$ are introduced in [16] where $H^{p}$ is the Hardy space.

\subsection{Fourier multipliers on modulation spaces}

In this section, we review the previous work about Fourier multipliers on modulation spaces. Then we introduce the main contribution of the present paper.

The Fourier multipliers $m(\xi)$ as an operator are defined in [14, 17-19] with

$$
T_{m} f(x):=\int_{\mathbb{R}^{n}} m(\xi) \mathcal{F} f(\xi) \mathrm{e}^{2 \pi \mathrm{i} x \cdot \xi} \mathrm{d} \xi
$$

where $2 \pi$ is unessential and the Fourier transform is normalised to be

$$
\hat{f}=\mathscr{F} f:=\int_{\mathbb{R}^{n}} f(x) \mathrm{e}^{-2 \pi \mathrm{i} x \cdot \xi} \mathrm{d} x .
$$

The set of the Fourier multipliers from the function space $X$ to $Y$ is denoted with $\mathcal{M}(X, Y)$ as a subspace of the bounded linear operator space $L(X, Y)$ and $T: m \mapsto T_{m}=$ $m(D)$ is the embedding mapping. Fourier multipliers and the corresponding operators are seldom distinct. Notice $\mathcal{M}^{p, q}=\mathcal{M}\left(L^{p}, L^{q}\right)$ and denote $\mathcal{M}^{p}=\mathcal{M}^{p, p}, 1 \leq p \leq q \leq \infty$ [20-22].

The Fourier multipliers discussed in this paper generally do not preserve most of the Lebesgue spaces or even the Besov spaces (see $[18,23]$ ). This is the motivation to study the boundedness properties on other function spaces. The Fourier multipliers for the modulation spaces have been developed in many papers [14, 17, 24-26] where the so-called unimodular Fourier multipliers were studied and applied into PDEs. One of the most famous examples is $\mathrm{e}^{\mathrm{i}|\xi|^{2}} \in \mathcal{M}\left(M_{p, q}^{s}\right)$ as occurs in the Schrödinger equation.

Let us recall the main results relating with our study. Reference [14] (Theorem 1 as its main result) proved that $\mathrm{e}^{\mathrm{i}|\xi|^{\alpha}} \in \mathcal{M}\left(M_{p, q}^{s}\right), \alpha \in[0,2]$. Moreover, we have the following estimate of $\mathrm{e}^{\mathrm{i} t|\xi|^{2}}$ under the norm $\mathcal{M}\left(M_{p, q}^{s}\right)$ (see Corollary 5 in [14] and also [24, 27]):

$$
\|u(t)\|_{M_{p, q}} \lesssim\langle t\rangle^{\frac{n}{2}}\|f\|_{M_{p, q}}, \quad u(t)=\mathrm{e}^{\mathrm{i} t|D|^{2}} f .
$$

The case $\alpha>2$ is well discussed in [25] where $|\xi|^{\alpha}$ has been replaced with a real-valued homogeneous function of degree $\alpha$. 
Furthermore, [17] (Theorem 1 and Theorem 2) gave the following estimate:

$$
\left\|\mathrm{e}^{\mathrm{i} t|D|^{\alpha}} f\right\|_{M_{p, q}^{s}} \lesssim\langle t\rangle^{n\left|\frac{1}{2}-\frac{1}{p}\right|}\|f\|_{M_{p, q}^{s},}, \frac{1}{2}<\alpha \leq 2 .
$$

However, when $\alpha>2$, the multiplier loses a regularity $\gamma=(\alpha-2) n\left|\frac{1}{2}-\frac{1}{p}\right|$, precisely

$$
\left\|\mathrm{e}^{\mathrm{i} t|D|^{\alpha}} f\right\|_{M_{p, q}^{s-\gamma}} \lesssim\langle t\rangle^{n\left|\frac{1}{2}-\frac{1}{p}\right|} \mid f \|_{M_{p, q}^{s}, \quad \alpha>2}
$$

while the sharpness of the regularity lost is discussed in [25]. [13, 16] studied the Hardy type modulation spaces and have shown a similar estimate, namely

$$
\left\|\mathrm{e}^{\mathrm{i} t|D|^{\alpha}} f\right\|_{\mu_{p, q}^{s-\gamma}} \lesssim\langle t\rangle^{n\left|\frac{1}{2}-\frac{1}{p}\right|}\|f\|_{\mu_{p, q}^{s}}
$$

where $\gamma=n(\alpha-2)\left|\frac{1}{2}-\frac{1}{p}\right|$.

The purpose of the paper is to study the unimodular multipliers with a time parameter $m(\xi, t)=\mathrm{e}^{\mathrm{i} t p(\xi)}$ and to estimate

$$
C(t)=\left\|\mathrm{e}^{\mathrm{i} t(\xi)}\right\|_{\mathcal{M}\left(M_{p, q}, M_{p, q}^{s}\right)}
$$

where $p(\xi)$ is a real-valued function satisfying the Mihilin type condition

$$
\left|\partial^{\gamma} p(\xi)\right| \lesssim|\xi|^{\alpha-|\gamma|}
$$

where $\alpha$ is extended to $(0,+\infty)$ and $0<\delta<N$ for large $N$.

Denote $\mathfrak{M}^{s, p}=\mathcal{M}\left(M_{p, q}, M_{p, q}^{s}\right)$ where $s$ represents the regularity that the multiplier gains (or loses when $s<0$ ). The main result of this paper can be stated in the following form. It indeed contains most results of the previous work.

Theorem 1.1 If the real-valued function $p(\xi)$ mentioned above satisfies $\left|\partial^{\gamma} p(\xi)\right| \sim|\xi|^{\alpha-|\gamma|}$, then

$$
C(t)=\left\|\mathrm{e}^{\mathrm{i} t p(\xi)}\right\|_{\mathfrak{M}^{s, p}} \lesssim\langle t\rangle^{(n+2 \eta)\left|\frac{1}{2}-\frac{1}{p}\right|},
$$

where $s=\left\{\begin{array}{ll}0, & 0<\alpha \leq 2, \\ (2-\alpha) n \mid \frac{1}{2}-\frac{1}{p}, & \alpha>2\end{array}\right.$ and $0 \leq \eta \leq 1$ will be determined in Section 3 when $\alpha$ is small. (It was shown that $\eta=0$ when $\alpha>\frac{1}{2}$.)

It will be restated in Theorem 4.2 in more precise form. In Theorem 3.1 we estimate the lower bound that the $\eta$ can reach. Furthermore, we will discuss the sharpness of $s$ in Section 4.2, namely the maximum value for $s$ is $(2-\alpha) n\left|\frac{1}{2}-\frac{1}{p}\right|$ such that $\left\|\mathrm{e}^{\mathrm{i} t(\xi)}\right\|_{\mathfrak{M} s, p}$ is controlled by $C(t)$.

Simply stated, we will do three things mainly in the paper:

(i) refine $\eta$ so that $\left\|\mathrm{e}^{\mathrm{i} t(\xi)}\right\|_{\mathfrak{M}^{s, p}} \lesssim\langle t\rangle^{(n+2 \eta)\left|\frac{1}{2}-\frac{1}{p}\right|}$ when $\alpha$ is small,

(ii) discuss the sharpness of $s$ with a lemma,

(iii) show the lower bound of $\left\|\mathrm{e}^{\mathrm{it} t(\xi)}\right\|_{\mathfrak{M}^{s, p} \text {. }}$.

Feature (i) is the main contribution of the paper. We obtain the smallest value of $\eta$ among the related research. As far as this author knows, (iii) is seldom discussed in the previous 
works. Meanwhile, we propose a generic process that is different from previous papers, and which allows us to handle the estimate of the unimodular multipliers (to be compared with $[16,17])$.

\subsection{Notations and organisation}

Throughout this paper, $A(x) \lesssim B(x)$ means that there exists a positive constant $C$ such that $A(x) \leq C B(x)$ for all $x$ in an abstract space where $A, B$ are two non-negative functions in the space, while $A(x) \sim B(x)$ is used to denote $A(x) \lesssim B(x) \lesssim A(x)$. $x \ll 1$ means that $x \geq 0$ is less than a small number. $\sum_{k \in \mathbb{Z}^{n}}\left(\sup _{k \in \mathbb{Z}^{n}}\right)$ will be simply written as $\sum_{k}\left(\sup _{k}\right)$, if there is no ambiguity. $L^{p}\left(\mathbb{R}^{n}\right)$ is shortened as $L^{p}$, if $n$ is arbitrary and kept uniform. Notice that $p \geq 1$ throughout this paper. Finally, we define $\left|D^{\delta} f\right|=\sum_{|\gamma|=\delta}\left|\partial^{\gamma} f\right|$. In fact, $D^{\delta} f \sim\left\{\partial^{\gamma} f,|\gamma|=\delta\right\}$ is regarded as a vector. $\dot{H}^{s, p}$ denotes the homogeneous Sobolev space, and $\dot{H}^{s}=\dot{H}^{s, 2}$.

This paper is organised as follows. In Section 2, we mainly discuss the representation of the Fourier multipliers on the modulation spaces. In Section 3, we refine the bound of the part near 0 of the multipliers as the main contribution of the paper. In Section 4, we study the boundedness of the unimodular Fourier multipliers by oscillatory integral theory (Lemma 4.1). We also give the sharpness argument as regards the regularity lost by the multipliers and prove the lower bound. The result in the previous section is applied to the local well-posedness of the dispersive equations in Section 5.

\section{Representation of Fourier multipliers}

In this section, we will discuss the representation of Fourier multipliers that is the fundamental step for the estimate and shown in (4) and (5). Several well-known lemmata are listed as follows which will be used in the sequel.

Lemma 2.1 (Sobolev embedding [28]) $H^{s} \hookrightarrow L^{p}, \frac{1}{p}=\frac{1}{2}-\frac{s}{n}, \frac{s}{n}<\frac{1}{2}$.

Lemma 2.2 (Bernstein theorem [11]) Let $L>\frac{n}{2}$ be an integer, then

$$
\|f\|_{\mathcal{F} L^{1}} \lesssim\|f\|_{L^{2}}^{1-\frac{n}{2 L}}\|f\|_{\dot{H}^{L}}^{\frac{n}{2 L}}
$$

By checking the proof Proposition 1.11 in [11], for any $t>0$ we have

$$
\begin{aligned}
\|f\|_{\mathcal{F} L^{1}} & \lesssim \int_{|x|<t}|\mathcal{F} f| \mathrm{d} x+\int_{|x|>t}|\mathcal{F} f| \mathrm{d} x \\
& \lesssim t^{\frac{n}{2}}\|\mathcal{F} f\|_{L^{2}}+\left.\int_{|x|>t}|| x\right|^{-L} \mathcal{F}\left(D^{L} f\right) \mid \mathrm{d} x \\
& \lesssim t^{\frac{n}{2}}\|f\|_{L^{2}}+t^{\frac{n}{r}-L}\left\|D^{L} f\right\|_{\mathcal{F} L^{r^{\prime}}} \\
& \lesssim t^{\frac{n}{2}}\|f\|_{L^{2}}+t^{\frac{n}{r}-L}\left\|D^{L} f\right\|_{L^{r}},
\end{aligned}
$$

where $L>\frac{n}{r}, 1 \leq r \leq 2$ is an integer. From the Sobolev embedding theorem,

$$
\|f\|_{\mathcal{F} L^{1}} \lesssim t^{\frac{n}{2}}\|f\|_{L^{2}}+t^{\frac{n}{r}-L}\left\||\xi|^{s} D^{L} f\right\|_{L^{2}},
$$

where $\frac{1}{r^{\prime}}=\frac{1}{2}-\frac{s}{n}\left(\right.$ or $\left.s=\frac{n}{2}-\frac{n}{r^{\prime}}\right), 0 \leq s<\frac{n}{2}$.

By (2), it follows a generalised version of Bernstein's theorem that will be used in Remark 3.2. 
Lemma 2.3 Let $L>\frac{n}{2}$ be an integer, then

$$
\|f\|_{\mathcal{F} L^{1}} \lesssim\|f\|_{L^{r}}^{1-\frac{n}{r L}}\|f\|_{\dot{H}^{L, r}}^{\frac{n}{r L}}
$$

where $1 \leq r \leq 2, r L>n$.

Lemma 2.4 $\mathcal{F} L^{1} \hookrightarrow \mathcal{M}^{p}$, for all $1 \leq p \leq \infty$. More precisely

$$
\left(\mathcal{F} L^{1}, L^{\infty}\right)_{\theta} \hookrightarrow \mathcal{M}^{p}, \quad \theta=2\left|\frac{1}{2}-\frac{1}{p}\right| .
$$

Lemma 2.5 (Nikol'skij-Triebel's inequality [11,22]) If $0<p \leq q \leq \infty, f \in L^{p}$ whose Fourier transform is supported in a compact subset $\Omega$ of $\mathbb{R}^{n}$, then $\left\|\square_{k} f\right\|_{q} \lesssim\|f\|_{p}$ uniformly for $k \in \mathbb{Z}^{n}$.

Now we assume that the multiplier $m$ is bounded from $M_{p_{1}, q_{1}}^{s_{1}}$ to $M_{p_{2}, q_{2}}^{s_{2}}, p_{1} \leq p_{2}$, namely

$$
\left(\sum_{k}\langle k\rangle^{s_{2} q_{2}}\left\|\mathcal{F}^{-1}\left(\psi_{k} m \mathcal{F} f\right)\right\|_{p_{2}}^{q_{2}}\right)^{\frac{1}{q_{2}}} \lesssim\left(\sum_{k}\langle k\rangle^{s_{1} q_{1}}\left\|\mathcal{F}^{-1}\left(\psi_{k} \mathcal{F} f\right)\right\|_{p_{1}}^{q_{1}}\right)^{\frac{1}{q_{1}}}
$$

If $f \in L^{p_{1}}, \operatorname{supp} \hat{f} \subset \Omega$ where $\Omega$ is a compact subset of $\mathbb{R}^{n}$ then

$$
\Lambda_{\Omega}=\left\{k \in \mathbb{Z}^{n} \mid \Omega \cap \operatorname{supp} \psi_{k} \neq \emptyset\right\}
$$

has finitely many elements and

$$
f=\sum_{k \in \Lambda_{\Omega}} \mathcal{F}^{-1}\left(\psi_{k} \mathcal{F} f\right)
$$

and from Nikol'skij-Triebel's inequality,

$$
\begin{aligned}
\langle k\rangle^{s_{2}}\left\|\mathcal{F}^{-1}\left(\psi_{k} m \mathcal{F} f\right)\right\|_{p_{2}} & \lesssim \sum_{k \in \Lambda_{\Omega}}\langle k\rangle^{s_{1}}\left\|\mathcal{F}^{-1}\left(\psi_{k} \mathcal{F} f\right)\right\|_{p_{1}} \\
& \lesssim \sum_{k \in \Lambda_{\Omega}}\langle k\rangle^{s_{1}}\|f\|_{p_{1}} .
\end{aligned}
$$

Then we have

$$
\begin{aligned}
\left\|\mathcal{F}^{-1}(m \mathcal{F} f)\right\|_{p_{2}} & \lesssim \sum_{k \in \Lambda_{\Omega}}\left\|\mathcal{F}^{-1}\left(\psi_{k} m \mathcal{F} f\right)\right\|_{p_{2}} \\
& \lesssim \sum_{k_{1}, k_{2} \in \Lambda_{\Omega}}\left\langle k_{2}\right\rangle^{-s_{2}}\left\langle k_{1}\right\rangle^{s_{1}}\|f\|_{p_{1}} \\
& \lesssim\langle\xi\rangle^{s_{1}-s_{2}}\|f\|_{p_{1}}
\end{aligned}
$$

where $\xi \in \Omega$.

Replacing $f$ with $\mathcal{F}^{-1}\left(\psi_{k} \mathcal{F} f\right)$, we have

$$
\left\|\mathcal{F}^{-1}\left(\psi_{k} m \mathcal{F} f\right)\right\|_{p_{2}} \lesssim\langle k\rangle^{s_{1}-s_{2}}\|f\|_{p_{1}},
$$


which implies a representation of the multipliers on the modulation spaces,

$$
\mathcal{M}\left(M_{p_{1}, q_{1}}^{s_{1}}, M_{p_{2}, q_{2}}^{s_{2}}\right)=W\left(\mathcal{M}^{p_{1}, p_{2}}, \ell^{s_{2}-s_{1},+\infty}\right), \quad 1 \leq p_{1} \leq p_{2}, q_{1} \leq q_{2} .
$$

By this fact, we only need to study $\mathcal{M}\left(M_{p_{1}, q}, M_{p_{2}, q}^{s}\right)$, but in this paper we are only interested in the following class of multipliers.

Lemma 2.6 (also see [25]) For $1 \leq p \leq \infty$,

$$
\mathcal{M}\left(M_{p, q}, M_{p, q}^{s}\right)=W\left(\mathcal{M}^{p}, \ell^{s,+\infty}\right) .
$$

Denote $\mathfrak{M}^{s, p}=\mathcal{M}\left(M_{p, q}, M_{p, q}^{s}\right)$ for short. When $s=0$, it is written as $\mathfrak{M}^{p}$. The representation of multipliers on modulation spaces leads us to Hormander's multipliers.

Remark 2.1 When $p_{1} \leq p_{2}<1$, one only has

$$
W\left(\mathcal{M}^{p_{1}, p_{2}}, \ell^{s_{2}-s_{1},+\infty}\right) \hookrightarrow \mathcal{M}\left(M_{p_{1}, q_{1}}^{s_{1}}, M_{p_{2}, q_{2}}^{s_{2}}\right),
$$

where $\mathcal{M}^{p_{1}, p_{2}}=\left\{m \in \mathcal{S}^{\prime} \mid\|m(D) f\|_{L^{p_{2}}} \leq C(R)\|f\|_{L^{p_{1}}}, R<\infty\right.$ is the size of the support of $\left.\hat{f}\right\}$. One has to apply (6) in extending the results to Hardy type spaces.

Our proof is based on the previous work, especially [14, 17, 25]. By Lemma 2.4, Lemma 2.6 and the interpolation theory, we have

$$
\|m\|_{\mathfrak{M}^{s, p}} \leq \sup _{k}\left\{\langle k\rangle^{s}\left\|\psi_{k} m\right\|_{\mathcal{F}^{1}}^{2\left|\frac{1}{p}-\frac{1}{2}\right|}\right\} .
$$

It remains to calculate $\left\|\psi_{k} m\right\|_{\mathcal{F}_{L^{1}}}$ for the estimate of $\|m\|_{\mathfrak{M}^{s, p}, \text { while the Bernstein theorem }}$ suggests calculating $\left\|\psi_{k} m\right\|_{\dot{H}^{L}}$. Thus the primary task is to calculate $\partial^{\gamma} \psi(\xi) \mathrm{e}^{\mathrm{i} t p(\xi)}$.

It is easy to see that

$$
\partial^{\gamma} \mathrm{e}^{\mathrm{i} t p(\xi)}=\mathrm{e}^{\mathrm{i} t p(\xi)} \sum_{m \leq|\gamma|} t^{m} \sum_{\sum_{i} \beta_{i}=\gamma} C_{\beta_{1} \cdots \beta_{m}} \partial^{\beta_{1}} p \cdots \partial^{\beta_{m}} p,
$$

which is a polynomial about $t$ multiplied by $\mathrm{e}^{\mathrm{i} t p(\xi)}$ with functional coefficients; then

$$
\partial^{\gamma} \phi(\xi) \mathrm{e}^{\mathrm{i} t p(\xi)}=\mathrm{e}^{\mathrm{i} t p(\xi)} \sum_{m \leq|\gamma|} t^{m} \sum_{\substack{\sum_{i} \beta_{i} \leq \gamma \\\left|\beta_{i}\right|>0}} \phi_{\beta_{1} \cdots \beta_{m}} \partial^{\beta_{1}} p \cdots \partial^{\beta_{m}} p,
$$

where $\phi, \phi_{\beta_{1} \cdots \beta_{m}} \in$ \&. Equation (8) yields

$$
\left|D^{L} \phi(\xi) \mathrm{e}^{\mathrm{i} t p(\xi)}\right| \lesssim \sum_{m \leq L}|t|^{m} \sum_{\substack{m \leq\left|\sum_{i} \beta_{i}\right| \leq L \\\left|\beta_{i}\right|>0}}\left|\partial^{\beta_{1}} p \cdots \partial^{\beta_{m}} p\right|,
$$

where $\phi \in$ s. Equations (9) and (8) will be applied in estimating the multipliers. With the assumption $\partial^{\gamma} p(\xi) \lesssim|\xi|^{\alpha-|\gamma|},|\gamma|>0$, it follows from (9) that

$$
\left|D^{L} \phi(\xi) \mathrm{e}^{\mathrm{i} t p(\xi)}\right| \lesssim\langle t\rangle^{L}|\xi|^{\alpha-L}
$$

This implies the basic thought of the paper (see Lemma 3.1 and Remark 3.2). 


\section{Near the origin}

We shall show the estimates of $\left\|\psi_{k} m\right\|_{\mathcal{F} L^{1}}$ by distinguishing between the cases when $k$ near the origin and $k$ near infinity. In this section, we focus on the former.

The following lemma generalises Lemma 9 in [17] as the main contribution of the paper. It shows a more precise upper bound.

Lemma 3.1 If $p \in C^{L}\left(\mathbb{R}^{n} \backslash\{0\}\right), L=\left[\frac{n}{2}\right]+1,\left|\partial^{\gamma} p(\xi)\right| \lesssim|\xi|^{\alpha-|\gamma|}$ for $0<|\gamma| \leq L$ and $\phi \in C_{0}^{\infty}$, then

$$
\left\|\phi(\xi) \mathrm{e}^{\mathrm{i} t p(\xi)}\right\|_{\mathcal{F} L^{1}} \lesssim \begin{cases}\langle t\rangle^{\frac{n}{2}}, & \alpha>\frac{1}{n+1}, 2 \nmid n \text { or } \alpha>\frac{2}{n+2}, 2 \mid n \\ \langle t\rangle^{\frac{n}{2}+\eta}, & \text { otherwise }\end{cases}
$$

where $\eta \in[0,1)$ is selected corresponding to $\alpha$.

$L$ is assumed to be large, but we will see that $L$ can be chosen as small as $\left[\frac{n}{2}\right]+1$.

Proof As in [17], from (9) we get

$$
\left|D^{L} \phi(\xi) \mathrm{e}^{\mathrm{i} t p(\xi)}\right| \lesssim\langle t\rangle^{L}|\xi|^{\alpha-L} \tilde{\phi}(\xi)
$$

Consider the following two cases.

Case 1: $\alpha>1$ or $\alpha>\frac{1}{2}$ when $n$ is odd. With Lemma 2.2 and (10), it follows that

$$
C(t):=\left\|\phi(\xi) \mathrm{e}^{\mathrm{i} t p(\xi)}\right\|_{\mathcal{F} L^{1}} \lesssim\langle t\rangle^{\frac{n}{2}}\left\||\xi|^{\alpha-L} \tilde{\phi}(\xi)\right\|_{2}^{\frac{n}{2 L}}
$$

where $\tilde{\phi} \in C_{C}^{\infty}$. Take $L=\left[\frac{n}{2}\right]+1$. whenever $\alpha>1$ or $\alpha>\frac{1}{2}$ if $n$ is odd, $L-\alpha<\frac{n}{2}$, so $|\xi|^{\alpha-L} \tilde{\phi}(\xi)$ must be square integrable.

Case 2: $\alpha \leq 1$. Decompose (8) as a polynomial into

$$
\begin{cases}H:=\mathrm{e}^{\mathrm{i} t p(\xi)} t^{L} P(\xi), & \text { the leading term, } \\ R:=\mathrm{e}^{\mathrm{i} t p(\xi)} \sum_{m<L} t^{m} P_{m}(\xi), & \text { the lower terms. }\end{cases}
$$

We have $\partial^{\gamma} \psi(\xi) \mathrm{e}^{\mathrm{i} t p(\xi)}=R+H$, and near 0

$$
|H| \lesssim t^{L}|\xi|^{L(\alpha-1)}, \quad|R| \lesssim t^{L-1}|\xi|^{\alpha-L}
$$

Unlike [16], we go back to the second step of (2). For any $\bar{t}>0$ we have

$$
\begin{aligned}
C(t) & \lesssim \bar{t}^{\frac{n}{2}}+\left.\int_{|x|>\bar{t}}|| x\right|^{-L} \mathcal{F} R\left|\mathrm{~d} x+\int_{|x|>\bar{t}}\right||x|^{-L} \mathcal{F} H \mid \mathrm{d} x \\
& \lesssim \bar{t}^{\frac{n}{2}}+\bar{t}^{\frac{n}{r_{1}}-L}\left\||\xi|^{s_{1}} R\right\|_{L^{2}}+\bar{t}^{\frac{n}{r_{2}}-L}\left\||\xi|^{s_{2}} H\right\|_{L^{2}} \\
& \lesssim \bar{t}^{\frac{n}{2}}+I_{1}+I_{2} .
\end{aligned}
$$

Take $\bar{t}=\langle t\rangle$. For the lower terms, we have

$$
I_{1} \lesssim\langle t\rangle^{\frac{n}{r_{1}}-1}\left\||\xi|^{\alpha-L+s_{1}} \tilde{\phi}(\xi)\right\|_{2} .
$$


To make it finite, we have to find $1<r_{1} \leq 2$ and an integer $L>\frac{n}{r_{1}}$ satisfying

$$
\left\{\begin{array}{l}
\frac{1}{r_{1}^{\prime}}=\frac{1}{2}-\frac{s_{1}}{n}, \quad 0 \leq s_{1}<\frac{n}{2} \\
L-\alpha-s_{1}<\frac{n}{2}
\end{array}\right.
$$

For the leading term, we have

$$
I_{2} \lesssim\langle t\rangle^{\frac{n}{r_{2}}}\left\||\xi|^{L(\alpha-1)+s_{2}} \tilde{\phi}(\xi)\right\|_{2}
$$

As above, we have to find $1<r_{2} \leq 2$ and $L>\frac{n}{r_{2}}$ so that

$$
\left\{\begin{array}{l}
\frac{1}{r_{2}^{\prime}}=\frac{1}{2}-\frac{s_{2}}{n}, \quad 0 \leq s_{2}<\frac{n}{2}, \\
L(1-\alpha)-s_{2}<\frac{n}{2}
\end{array}\right.
$$

Equations (13) and (14) are equivalent to

$$
\left\{\begin{array}{l}
L-\alpha<\frac{n}{r_{1}}, \\
L(1-\alpha)<\frac{n}{r_{2}}, \\
L \in \mathbb{Z}>\frac{n}{r_{1}}, \frac{n}{r_{2}}, \\
\frac{n}{2} \leq \frac{n}{r_{1}}, \frac{n}{r_{2}}<n,
\end{array}\right.
$$

where $\frac{n}{r_{1}}, \frac{n}{r_{2}}$ are both regarded as two variables. The mission is to find a solution $\left(L, \frac{n}{r_{1}}, \frac{n}{r_{2}}\right)$ to (15) such that $\frac{n}{r_{1}}, \frac{n}{r_{2}}$ are small enough, and then

$$
C(t) \lesssim\langle t\rangle^{\max \left\{\frac{n}{r_{1}}-1, \frac{n}{r_{2}}\right\}}
$$

Obviously, if $n$ is odd and $\alpha>\frac{1}{n+1}$, then $\left(L, \frac{n}{r_{1}}, \frac{n}{r_{2}}\right)=\left(\left[\frac{n}{2}\right]+1,\left[\frac{n}{2}\right]+1, \frac{n}{2}\right)$ is a solution, while if $n$ is even and $\alpha>\frac{2}{n+2}$, then $\left(L, \frac{n}{r_{1}}, \frac{n}{r_{2}}\right)=\left(\left[\frac{n}{2}\right]+1,\left[\frac{n}{2}\right]+1, \frac{n}{2}\right)$ is a solution. Now we try to find a solution on $\frac{n}{r_{1}}=\frac{n}{r_{2}}$. Since $L(1-\alpha) \leq L-\alpha$, it is only need to consider (13) that is equivalent to $1-\left\{\frac{n}{r_{1}}\right\}<\alpha$. It is easy to see that there exists $\frac{n}{2} \leq \frac{n}{r_{1}}<\left[\frac{n}{2}\right]+1$ satisfying the inequality, since $1-\left\{\frac{n}{r_{1}}\right\}$ runs through $\left(0, \frac{1}{2}\right)$ at least whenever $n$ is odd or even. This implies the following result:

$$
C(t) \lesssim \begin{cases}\langle t\rangle^{\frac{n}{2}}, & \alpha>\frac{1}{n+1}, 2 \nmid n \text { or } \alpha>\frac{2}{n+2}, 2 \mid n, \\ \langle t\rangle^{\frac{n}{2}+\eta}, & \text { otherwise, }\end{cases}
$$

where

$$
\eta= \begin{cases}\frac{1}{2}-\alpha+, & 2 \nmid n \\ 1-\alpha+, & 2 \mid n .\end{cases}
$$

As a result, $\frac{n}{r_{1}}, \frac{n}{r_{2}}$ can be restrained on $\frac{n}{2} \leq \frac{n}{r_{2}} \leq \frac{n}{r_{1}}<\left[\frac{n}{2}\right]+1$ and

$$
L=\left[\frac{n}{2}\right]+1
$$


Figure 1 To find the optimistic $\boldsymbol{\eta}$. Two solid lines are $y=x$ and $y=(1-\alpha)(x+\alpha)$, respectively, whose intersection point is $(1-\alpha, 1-\alpha)$. Note $x_{0}<\left[\frac{n}{2}\right]+1$ $-\alpha$. As is shown, we only need consider the grey domain.

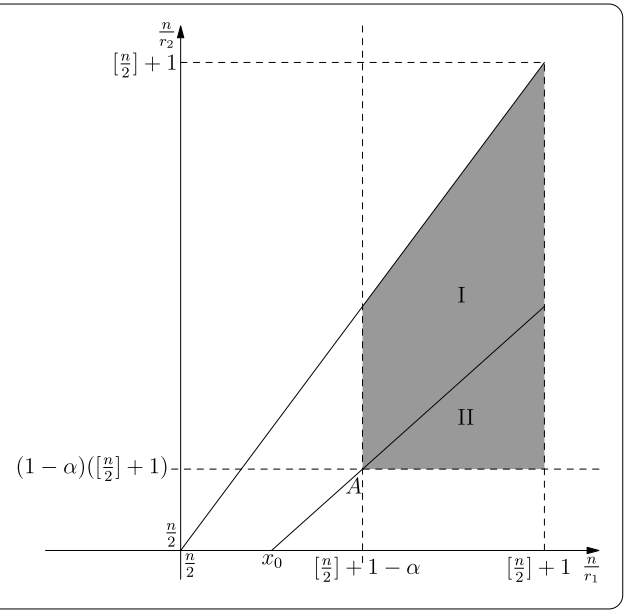

As is illustrated by Figure 1, in domain I, (15) is reduced to (13), while in domain II, it is reduced to

$$
\left\{\begin{array}{l}
L(1-\alpha)<\frac{n}{r_{2}}, \\
L \in \mathbb{Z}>\frac{n}{r_{1}}, \\
\frac{n}{2} \leq \frac{n}{r_{1}}, \frac{n}{r_{2}}<n,
\end{array}\right.
$$

which implies $\frac{n}{r_{2}}>(1-\alpha)\left(\left[\frac{n}{r_{1}}\right]+1\right)$.

Noticing that $I_{1} \lesssim\langle t\rangle^{\frac{n}{2}}$, we will only focus on $I_{2}$, i.e. $r_{2}$. Figure 1 shows that the point $A\left(\left[\frac{n}{2}\right]+1-\alpha,(1-\alpha)\left(\left[\frac{n}{2}\right]+1\right)\right)$ is the optimal one. Now (16) can be modified as

$$
C(t) \lesssim \begin{cases}\langle t\rangle^{\frac{n}{2}}, & \alpha>\frac{1}{n+1}, 2 \nmid n \text { or } \alpha>\frac{2}{n+2}, 2 \mid n\left(\text { esp. } \alpha>\frac{1}{2}\right), \\ \langle t\rangle^{\frac{n}{2}+\eta}, & \text { otherwise, }\end{cases}
$$

where

$$
\eta= \begin{cases}\frac{1}{2}-\frac{n+1}{2} \alpha+, & 2 \nmid n, \\ 1-\frac{n+2}{2} \alpha+, & 2 \mid n,\end{cases}
$$

which is determined by the coordinates of point $A$.

Remark 3.1 If we take $\bar{t}=\langle t\rangle^{\beta}$ where $\frac{n}{2} \beta=\beta\left(\frac{n}{r_{2}}-L\right)+L, \beta>1$, then $\eta=\frac{n}{2}(\beta-1)$ can be smaller. Thus one can see

$$
\eta= \begin{cases}\frac{1}{2}-\frac{n+1}{2} \alpha, & 2 \nmid n, \\ 1-\frac{n+2}{2} \alpha, & 2 \mid n .\end{cases}
$$

Remark 3.2 We introduce another approach which is easier but less accurate. Applying Lemma 2.3, we have

$$
\left\|\phi(\xi) \mathrm{e}^{\mathrm{i} t p(\xi)}\right\|_{\mathcal{F} L^{1}} \lesssim\langle t\rangle^{\frac{n}{r}}\left\||\xi|^{\alpha-L} \tilde{\phi}(\xi)\right\|_{r}^{\frac{n}{r L}},
$$


where $1 \leq r \leq 2, r L>n, \tilde{\phi} \in C_{C}^{\infty}$. To ensure $|\xi|^{\alpha-L} \tilde{\phi}(\xi) \in L^{r}$, we choose $1 \leq r \leq 2$ satisfying

$$
\left\{\begin{array}{l}
r(L-\alpha)<n, \\
L=\left[\frac{n}{r}\right]+1,
\end{array} \quad \text { namely } \alpha>1-\left\{\frac{n}{r}\right\} .\right.
$$

Obviously there exists such $r$ and a real number $\eta:=\frac{n}{r}-\frac{n}{2}>\frac{1}{2}-\alpha$. Then

$$
\left\|\phi(\xi) \mathrm{e}^{\mathrm{i} t p(\xi)}\right\|_{\mathcal{F} L^{1}} \lesssim\langle t\rangle^{\frac{n}{2}+\eta}, \quad 0 \leq \eta<1 .
$$

We still have $\eta=0$ when $\alpha>1-\left\{\frac{n}{2}\right\}$ as (16).

Remark 3.3 The inequality (9) is the key to the proof. Actually, given any multiplier $\partial^{\gamma} m(\xi, t)=H+R, m(\xi, t) \in L_{t}^{\infty} L_{\text {loc }, \xi}^{2}$ satisfying

$$
|H| \lesssim\langle t\rangle^{|\gamma|}|\xi|^{|\gamma|(\alpha-1)}, \quad|R| \lesssim\langle t\rangle^{|\gamma|-1}|\xi|^{\alpha-|\gamma|}, \quad \xi \text { is near } 0, \alpha>0
$$

which yields $\left|D^{L} m(\xi, t)\right| \lesssim\langle t\rangle^{L}|\xi|^{\alpha-L}$, and we have the same result.

Remark 3.4 A simple example is $p(\xi)=|\xi|^{\alpha}\left(\right.$ or $\left.\langle\xi\rangle^{\alpha}\right)$. It occurs in the wave equation $(\alpha=1)$ or the Schrödinger equation $(\alpha=2)$.

\section{Near infinity}

In this section we come to the infinite case with oscillatory integrals. The sharpness and the lower bound will be discussed in the second subsection.

\subsection{Oscillatory integrals}

By Lemma 2 in [25] (also see [14]), we have

$$
\left\|\psi_{k}(\xi) \mathrm{e}^{\mathrm{i} t|\xi|^{2}}\right\|_{\mathcal{F}^{1}}=\left\|\psi(\xi) \mathrm{e}^{\mathrm{i} t|\xi|^{2}}\right\|_{\mathcal{F} L^{1}} .
$$

Thus we can state the classical result here that

$$
\left\|\mathrm{e}^{\mathrm{i} t|\xi|^{2}}\right\|_{\mathfrak{M}^{p}} \lesssim\langle t\rangle^{n\left|\frac{1}{2}-\frac{1}{p}\right|}
$$

This technique makes a linear alteration of the phase without affecting the norm. More generally,

$$
\left\|\psi_{k}(\xi) \mathrm{e}^{\mathrm{i} t t_{(\xi)}}\right\|_{\mathcal{F} L^{1}}=\left\|\psi(\xi) \mathrm{e}^{\mathrm{i} t q_{k}(\xi)}\right\|_{\mathcal{F} L^{1}}
$$

where $q_{k}(\xi):=p(\xi+k)-p(k)-\nabla p(k) \cdot \xi$ and we have (see Lemma 3.3 in [25] and Lemma 2 in [14])

$$
\left|\partial^{\gamma} q_{k}(\xi)\right| \lesssim|k|^{\alpha-2}, \quad \xi \in \operatorname{supp} \psi
$$

Equations (21) and (9) imply that for large $k$ and $\alpha>1$

$$
\left\|\psi_{k}(\xi) \mathrm{e}^{\mathrm{i} t p(\xi)}\right\|_{\mathcal{F} L^{1}} \lesssim\langle t\rangle^{\frac{n}{2}}\left\||k|^{L(\alpha-2)} \tilde{\psi}(\xi)\right\|_{2}^{\frac{n}{2 L}} \lesssim\langle t\rangle^{\frac{n}{2}}|k|^{\frac{n}{2}(\alpha-2)},
$$

where $\tilde{\psi} \in C_{0}^{\infty}$ has the same support as $\psi$. Therefore, we have the following results. 
Theorem 4.1 If $0<\alpha \leq 2$ with the assumption of Lemma 3.1, then

$$
\left\|\mathrm{e}^{\mathrm{i} t(\xi)}\right\|_{\mathfrak{M}^{p}} \lesssim\langle t\rangle^{(n+2 \eta)\left|\frac{1}{2}-\frac{1}{p}\right|}
$$

Proof It is true when $0<\alpha \leq 1$ obviously (consider (11) at infinity), while with (22) it holds in the case $1<\alpha \leq 2$.

Now it is sufficient to consider the case $\alpha>2$, for Theorem 4.1. Denote

$$
J_{k}(x)=\mathcal{F}^{-1}\left(\psi_{k} \mathrm{e}^{\mathrm{i} t p(\xi)}\right) \sim \int_{\mathbb{R}^{n}} \mathrm{e}^{\mathrm{i} \Phi(\xi, x, t, k)} \psi(\xi) \mathrm{d} \xi,
$$

where $\Phi(\xi, x, t, k)=t p(\xi+k)+x \cdot \xi$ and the implicative constant is unessential. Then $\left\|\psi_{k}(\xi) \mathrm{e}^{\mathrm{i} t p(\xi)}\right\|_{\mathcal{F} L^{1}}=\left\|J_{k}\right\|_{L^{1}}$.

We need the following lemma, which is due to Littman $[29,30]$ but has been refined.

Lemma 4.1 Let $\psi \in C_{C}^{\infty}$ be supported in $\Omega$. If $p \in C^{\infty}$ and its Hessian matrix Hp is nonsingular in $\Omega$, then there exists a large a such that

$$
\int_{\mathbb{R}^{n}} \mathrm{e}^{\mathrm{i} \Phi(\xi, x)} \psi(\xi) \mathrm{d} \xi \lesssim\langle\operatorname{det} H p\rangle^{-\frac{1}{2}}\left(1+\frac{|x|}{\langle\nabla p\rangle}\right)^{-a},
$$

where $\Phi(\xi, x)=p(\xi)+x \cdot \xi$

One also can get it with oscillatory integrals [31] considering the two cases $|x| \sim|\nabla p|$ and $|x| \nsim|\nabla p|$ separatively. Strictly speaking, it should be ensured that $\langle\operatorname{det} H p\rangle \lesssim\langle\nabla p\rangle$ for the inequality holds, and it indeed does in this context.

$\Phi_{k}(\xi, x, t)=t q_{k}(\xi)+x \cdot \xi$ does not have any critical point out of the domain $|x| \sim t|k|^{\alpha-2}$ where $q_{k}(\xi):=p(\xi+k)-p(k)-\nabla p(k) \cdot \xi$ defined as (20). With the classical argument of oscillatory integrals (see [31]) and replacing $p$ with $t q_{k}$ in Lemma 4.1, we have for $|k| \geq 1$

$$
\begin{aligned}
\left\|\mathrm{e}^{\mathrm{i} t p(\xi)} \psi_{k}(\xi)\right\|_{\mathcal{F} L^{1}} & =\left\|\mathrm{e}^{\mathrm{i} t q_{k}(\xi)} \psi(\xi)\right\|_{\mathcal{F} L^{1}} \\
& \sim \int_{|x| \sim t|k|^{\alpha-2}} \mathrm{~d} x\left|\int_{\mathbb{R}^{n}} \mathrm{e}^{\mathrm{i} \Phi_{k}(\xi, x, t)} \psi(\xi) \mathrm{d} \xi\right| \\
& \lesssim\langle t\rangle^{\frac{n}{2}}|k|^{\frac{n}{2}(\alpha-2)},
\end{aligned}
$$

where $p$ satisfies, additionally to the assumptions of Lemma 3.1, $\left|D^{2} p(\xi)\right| \sim|\xi|^{\alpha-2}$, and the Hessian matrix $H p$ is nonsingular on $\mathbb{R}^{n} \backslash\{0\}$. The last assumption yields

$$
\left|\operatorname{det} H q_{k}(\xi)\right| \sim\left|D^{2} q_{k}(\xi)\right| \sim\left|\nabla q_{k}(\xi)\right| \sim|k|^{\alpha-2}, \quad|\xi| \sim|k| .
$$

We have the following.

Theorem 4.2 If $p(\xi)$ from Lemma 3.1 satisfies $\left|D^{2} p(\xi)\right| \sim|\xi|^{\alpha-2}$ and the Hessian matrix Hp is nonsingular on $\mathbb{R}^{n} \backslash\{0\}$, then

$$
\left\|\mathrm{e}^{\mathrm{i} t(\xi)}\right\|_{\mathfrak{M}^{s, p}} \lesssim\langle t\rangle^{(n+2 \eta)\left|\frac{1}{2}-\frac{1}{p}\right|},
$$

where $s=\left\{\begin{array}{ll}0, & 0<\alpha \leq 2, \\ (2-\alpha) n\left|\frac{1}{2}-\frac{1}{p}\right|, & \alpha>2,\end{array}\right.$ and $\eta$ is the same as in Lemma 3.1. 
Proof The discussion above shows that for large $k$

$$
\left\|\psi_{k} \mathrm{e}^{\mathrm{i} t p(\xi)}\right\|_{\mathcal{M}^{p}} \lesssim\langle t\rangle^{n\left|\frac{1}{2}-\frac{1}{p}\right|}|k|^{-s}
$$

Then we combine this estimate with Lemma 3.1 to complete the proof.

Remark 4.1 An example for such $p(\xi) \in C^{L}\left(\mathbb{R}^{n} \backslash\{0\}\right)$ is the homogeneous function of degree $\alpha$.

\subsection{Sharpness and lower bound}

The multiplier will lose a regularity $s=(2-\alpha) n\left|\frac{1}{2}-\frac{1}{p}\right|$ when $\alpha>2$ in Theorem 4.2. To show an easy proof of the sharpness, we employ a lemma implicit in [25] as the key of the argument. In particular, we only need to estimate the upper bound of $\|\cdot\|_{\mathcal{F} L^{\infty}}$ to get the lower bound of $\|\cdot\|_{\mathcal{F} L^{1}}$.

\section{Lemma 4.2}

$$
\left\|\psi_{k} m\right\|_{\mathcal{F} L^{1}}\left\|\psi_{k} m\right\|_{\mathcal{F} L^{\infty}} \geq C(\psi)>0
$$

where $m$ is any Fourier multiplier whose inverse is $m^{-1}(\xi, t)=\overline{m(\xi, t)}$.

Proof Notice that $\mathcal{F} L^{1} \hookrightarrow \mathcal{M}^{p}, \mathcal{F} L^{\infty} \hookrightarrow \mathcal{M}^{p, p^{\prime}}, 1 \leq p<2$. With the interpolation theorems, we have

$$
\begin{aligned}
\left\|\psi_{k} m\right\|_{\mathcal{F} L^{1}}\left\|\psi_{k} m\right\|_{\mathcal{F} L^{\infty}} & \gtrsim\left\|\psi_{k} m^{-1}\right\|_{\mathcal{M}^{p, p}}^{\frac{1}{\theta}}\left\|\psi_{k} m\right\|_{\mathcal{M}^{p, p^{\prime}}}^{\frac{1}{\theta}} \\
& \gtrsim\left\|\psi_{k}^{2}\right\|_{\mathcal{M}^{p, p^{\prime}}}^{\frac{1}{\theta}} \\
& =C(\psi),
\end{aligned}
$$

where $\theta=2\left(\frac{1}{p}-\frac{1}{2}\right), 1 \leq p<2$ and $C(\psi)$ does not depend on $k$ or $t$.

On the other hand,

$$
\left\|\psi_{k} \mathrm{e}^{\mathrm{i} t p(\xi)}\right\|_{\mathcal{F} L^{\infty}} \lesssim|t|^{-\frac{n}{2}}|k|^{\frac{n}{2}(2-\alpha)}
$$

for Lemma 4.1. We obtain

$$
\left\|\psi_{k} \mathrm{e}^{\mathrm{i} t p(\xi)}\right\|_{\mathcal{F}^{1}} \sim\langle t\rangle^{\frac{n}{2}}|k|^{\frac{n}{2}(\alpha-2)}
$$

where $p(\xi)$ is a function as in Theorem 4.2. Equation (26) implies the sharpness of $s$ in the case $\alpha>2$. Replacing $\mathcal{F} L^{1}$ with $\mathcal{M}^{p}$ in Lemma 4.2, we get the lower bound

$$
\left\|\psi_{k} \mathrm{e}^{\mathrm{i} t p(\xi)}\right\|_{\mathcal{M}^{p}} \gtrsim\left(\langle t\rangle^{n}|k|^{n(\alpha-2)}\right)^{\left|\frac{1}{2}-\frac{1}{p}\right|},
$$

which yields

$$
\left\|\mathrm{e}^{\mathrm{i} t p(\xi)}\right\|_{\mathfrak{M}^{s, p}} \gtrsim\langle t\rangle^{n\left|\frac{1}{2}-\frac{1}{p}\right|}
$$

When $\alpha=2$, we have $\left\|\mathrm{e}^{\mathrm{i} t p(\xi)}\right\|_{\mathfrak{M}^{p}} \sim\langle t\rangle^{n\left|\frac{1}{2}-\frac{1}{p}\right|}$. However, (27) will not be applied to the Cauchy problem. 
Remark 4.2 Reference [25] provided an alternative method for the sharpness argument. It can be summarised in the following steps:

(i) establish the identity $m(D) U_{\lambda}=U_{\lambda} m(\lambda D)$ or $m(\lambda D)=U_{\frac{1}{\lambda}} m(D) U_{\lambda}$,

(ii) calculate $\|m(\lambda D)\| \lesssim \lambda^{s^{\prime}}$ with the identity for $s^{\prime}>0$,

(iii) estimate $\|m(\lambda D)\| \gtrsim \lambda^{s}$ with oscillatory integrals,

where $U_{\lambda} f(x)=f(\lambda x)$. Combining the results listed above, we conclude $s \leq s^{\prime}$. Notice that the dilation properties of modulation spaces are employed in the step (ii). Generally, it is advocated to find a one-parameter group $\rho(\lambda)$ acting on multipliers so that

$$
\lambda^{s} \lesssim\|(\rho(\lambda) m)(D)\| \lesssim \lambda^{s^{\prime}}
$$

Remark 4.3 (25) is also the key to get the Strichartz estimates since $\mathcal{F} L^{\infty} \hookrightarrow \mathcal{M}^{p, p^{\prime}}$.

\section{Application to Cauchy problem}

Theorem 4.2 provides the time-space estimate [32] for the linear dispersive equations, namely

$$
\|u(t)\|_{M_{p, q}^{s}} \lesssim C(t)\left\|u_{0}\right\|_{M_{p, q}}
$$

where $u(t)=\mathrm{e}^{\mathrm{i} t p(D)} u_{0}$ and $C(t)$ is estimated by Theorem 4.2. When $s=0,\left\{\mathrm{e}^{\mathrm{i} t p(D)}\right\}$ forms a $C_{0}$ semigroup named the dispersive semigroup.

Now we consider the Cauchy problem for the nonlinear dispersive equation,

$$
\left\{\begin{array}{l}
\mathrm{i} \partial_{t} u-p(D) u+F(u)=0, \\
u(0, x)=u_{0}(x)
\end{array}\right.
$$

where the complex variable function $F(u)=\lambda|u|^{2 k} u$. If the initial condition is $u\left(t_{0}, x\right)=$ $u_{0}(x)$, we have $u(t, x)=v\left(t-t_{0}, x\right)$ where $v$ is the solution to (28). The Cauchy problem can be written in an equivalent integral form,

$$
u(t)=\mathrm{e}^{\mathrm{i} t p(D)} u_{0}-i \int_{0}^{t} \mathrm{e}^{\mathrm{i}(t-\tau) p(D)} F(u(\tau)) \mathrm{d} \tau
$$

In this section, we shall apply the estimate of the multipliers into the local well-posedness of the equation on the modulation spaces. Define two operators on $X=C\left([0, T], M_{p, 1}^{s}\right)$ :

$$
\begin{aligned}
& \mathcal{T} u:=\mathrm{e}^{\mathrm{i} t p(D)} u_{0}-i \int_{0}^{t} \mathrm{e}^{\mathrm{i}(t-\tau) p(D)} F(u(\tau)) \mathrm{d} \tau, \\
& \mathcal{A} u:=\int_{0}^{t} \mathrm{e}^{\mathrm{i}(t-\tau) p(D)} F(u(\tau)) \mathrm{d} \tau .
\end{aligned}
$$

Assuming $\alpha \leq 2$, we immediately have

$$
\begin{aligned}
& \|\mathcal{T} u\|_{X} \leq C(T)\left(\left\|u_{0}\right\|_{M_{p, 1}^{s}}+T\|F(u)\|_{X}\right), \\
& \|\mathcal{T} u-\mathcal{T} v\|_{X} \leq C(T) T\|F(u)-F(v)\|_{X},
\end{aligned}
$$


where $C(t) \sim\left\|\mathrm{e}^{\mathrm{i} t(\xi)}\right\|_{\mathfrak{M}^{p}}$. Restrained to the ball $B_{R}=\left\{\|u\|_{X} \leq R\right\}$,

$$
\left\{\begin{array}{l}
\|\mathcal{T} u\|_{X} \leq C(T)\left(\left\|u_{0}\right\|_{M_{p, 1}^{s}}+T R^{2 k+1}\right), \\
\|\mathcal{T} u-\mathcal{T} v\|_{X} \leq C(T) T R^{2 k}\|u-v\|_{X} .
\end{array}\right.
$$

With (29), we obtain a quantitative form of the local well-posedness for (28) (see [17]).

Corollary 5.1 Assume $p$ is the same as in Theorem 4.2 and $0<\alpha \leq 2$. If

$$
\left\|u_{0}\right\|_{M_{p, 1}^{s}} \leq C\langle T\rangle^{-(n+2 \eta)\left|\frac{1}{2}-\frac{1}{p}\right|\left(1+\frac{1}{2 k}\right)-\frac{1}{2 k}},
$$

then (28) has a unique solution $u \in C\left([0, T], M_{p, 1}^{s}\right)$ where the constant $C$ depends on $k$ and $n$.

Proof For (29), $\mathcal{T}$ is a contraction mapping on $B_{R}$, if $R$ satisfies

$$
\left\{\begin{array}{l}
C(T)\left(\left\|u_{0}\right\|_{M_{p, 1}^{s}}+T R^{2 k+1}\right) \leq R \\
C(T) T R^{2 k} \leq \frac{1}{2}
\end{array}\right.
$$

Now (30) suffices for the previous two inequalities to hold.

Remark 5.1 The assumption $\alpha \leq 2$ is indispensable for Theorem 4.2 (see [25]). $F(u)$ can be chosen as $\mathrm{e}^{\rho|u|^{2 k}} u$. Then $R^{2 k}$ will be replaced with $\mathrm{e}^{\rho R^{2 k}}$ in (29). The key point is that $F$ has Lipschitz type property. Note that $\eta=0$ when $\alpha>\frac{1}{2}$ as is shown in the literature.

Remark 5.2 The results can be generalised to $\alpha$-modulation spaces analogically where one should consider the so-called $\alpha$-covering rather than the uniform decomposition. See [33] for the basic concepts.

Remark 5.3 Our method can also be applied in the estimate of the multipliers on Hardy type modulation spaces where we shall use $\mathcal{F} L^{p}, 0<p<1$ rather than $\mathcal{F} L^{1}$. First of all, one should return to Remark 2.1, then use Nikol'skij-Triebel's inequality (see Lemma 14 in [16]), say $\mathcal{F} L^{p} \hookrightarrow \mathcal{M}^{p, p}$. Then one employs the following Bernstein type theorem: if integer $L>n\left(\frac{1}{p}+\frac{1}{r}-1\right)$, then

$$
\|f\|_{\mathcal{F} L^{p}} \lesssim\|f\|_{L^{q}}^{1-\theta}\|f\|_{\dot{H}^{L, r}}^{\theta}
$$

where $\theta=\frac{n(1 / p+1 / q-1)}{L+n(1 / q-1 / r)}, \frac{1}{2} \vee\left(1-\frac{1}{p}\right) \leq \frac{1}{r}, \frac{1}{q} \leq 1$. In the particular case when $q=r$,

$$
\|f\|_{\mathcal{F} L^{1}} \lesssim\|f\|_{L^{r}}^{1-\frac{n}{L}\left(\frac{1}{p}+\frac{1}{r}-1\right)}\|f\|_{\dot{H}^{L, r}}^{\frac{n}{L r}}, \quad \frac{1}{2} \vee\left(1-\frac{1}{p}\right) \leq \frac{1}{r}, \frac{1}{q} \leq 1
$$

Once again, it leads to the partial derivatives of multipliers. 


\section{Acknowledgements}

This work is partially supported by NSF of China (Grant No. 11271330, 10931001) and NSFZJ of China (Grant No. Y604563). The author wishes to thank Professor Fan who checked the whole article.

\section{Received: 29 October 2013 Accepted: 6 January 2014 Published: 27 Jan 2014}

\section{References}

1. Cordero, E, Nicola, F: Sharpness of some properties of Wiener amalgam and modulation spaces. Bull. Aust. Math. Soc. 80, 105-116 (2009)

2. Feichtinger, HG: Modulation spaces on locally compact Abelian group. Technical Report. University of Vienna, Vienna (1983). Also in Wavelets and Their Applications, pp. 99-140. Allied Publishers (2003)

3. Feichtinger, HG: Modulation spaces: looking back and ahead. Sampl. Theory Signal Image Process., Int. J. 5, 109-140 (2006)

4. Kobayashi, M: Modulation spaces $M^{p, q}$ for $0<p, q \leq \infty$. J. Funct. Spaces Appl. 4(3), 329-341 (2006)

5. Kobayashi, M: Dual of modulation spaces. J. Funct. Spaces Appl. 5(1), 1-8 (2007)

6. Kobayashi, M, Sugimoto, M: The inclusion relation between Sobolev and modulation spaces. J. Funct. Anal. 260, 3189-3208 (2011)

7. Okoudjou, KA: Embeddings of some classical Banach spaces into modulation spaces. Proc. Am. Math. Soc. 132(6), 1639-1647 (2004)

8. Sugimoto, $\mathrm{M}$, Tomita, $\mathrm{N}$ : The dilation property of modulation spaces and their inclusion relation with Besov spaces. J. Funct. Anal. 248, 79-106 (2007)

9. Tomita, N: On the Hörmander multiplier theorem and modulation spaces. Appl. Comput. Harmon. Anal. 26, 408-415 (2009)

10. Triebel, H: Modulation spaces on the Euclidean n-space. Z. Anal. Anwend. 2(5), 443-457 (1983)

11. Wang, BX, Huo, ZH, Hao, CC, Guo, ZH: Harmonic Analysis Method for Nonlinear Evolution Equations. World Scientific, Singapore (2011)

12. Gröchenig, K: Foundations of Time-Frequency Analysis. Birkhäuser, Boston (2001)

13. Chen, JC, Zhong, Y: Modulation space estimates for the fractional integral operators. Sci. China Math. 54(7), 1478-1489 (2011)

14. Bényi, A, Grochenig, K, Okoudjou, KA, Rogers, LG: Unimodular Fourier multipliers for modulation spaces. J. Funct. Anal. 246, 366-384 (2007)

15. Feichtinger, HG: Generalized amalgams, with applications to Fourier transform. Can. J. Math. 42, 395-409 (1990)

16. Chen, JC, Fan, DS, Sun, LJ, Zhang, CJ: Estimates for unimodular multipliers on modulation Hardy spaces. J. Funct. Spaces Appl. (2013, in press)

17. Chen, JC, Fan, DS, Sun, LJ: Asymptotic estimates for unimodular Fourier multipliers on modulation spaces. Discrete Contin. Dyn. Syst. 32(2), 467-485 (2012)

18. Mizuhara, T: On Fourier multipliers of homogeneous Besov spaces. Math. Nachr. 133, 155-161 (1994)

19. Hirschman, II: On multiplier transformations. Duke Math. J. 26, 211-242 (1959)

20. Maz'ya, V, Shaposhnikova, TO: Theory of Sobolev Multipliers: With Applications to Differential and Integral Operators. Springer, Berlin (2011)

21. Grafakos, L: Classic and Modern Fourier Analysis. Prentice Hall, New York (2003)

22. Triebel, $\mathrm{H}$ : Theory of Function Spaces. Birkhäuser, Basel (1983)

23. Hörmander, L: Estimates for translation invariant operators in $L^{P}$-spaces. Ann. Math. 104, 93-140 (1960)

24. Bényi, A, Okoudjou, KA: Local well-posedness of nonlinear dispersive equations on modulation spaces. Bull. Lond. Math. Soc. 41, 549-558 (2009)

25. Miyachi, A, Nicola, F, Rivetti, S, Tabacco, A, Tomita, N: Estimates for unimodular Fourier multipliers on modulation space. Proc. Am. Math. Soc. 137, 3869-3883 (2009)

26. Feichtinger, HG, Narimani, G: Fourier multipliers of classical modulation spaces. Appl. Comput. Harmon. Anal. 21(3), 349-359 (2006)

27. Wang, B, Zhao, L, Boling, G: Isomeric decomposition operators, function spaces $E_{p, q}^{\lambda}$ and applications to nonlinear evolution equations. J. Funct. Anal. 233(1), 1-39 (2006)

28. Hörmander, L: The Analysis of Linear Partial Differential Operators, vol. 3. Springer, Berlin (1985)

29. Domar, Y: On the spectral synthesis problem for $(n-1)$-dimensional of $\mathbb{R}^{n}, n \leq 2$. Ann. Math. 9, 23-37 (1971)

30. Littman, W: Fourier transforms of surface-carried measures and differentiability of surface averages. Bull. Am. Math. Soc. 69, 766-770 (1963)

31. Stein, EM: Harnonic Analysis. Princeton University Press, Princeton (1993)

32. Cordero, E, Nicola, F: Some new Strichartz estimates for the Schrödinger equation. J. Differ. Equ. 245, 1945-1974 (2008)

33. Borup, L, Nielsen, M: Boundedness for pseudodifferential operators on multivariate $\alpha$-modulation space. Ark. Mat. 44 241-259 (2006)

10.1186/1029-242X-2014-43

Cite this article as: Song: Unimodular Fourier multipliers with a time parameter on modulation spaces. Journal of Inequalities and Applications 2014, 2014:43 